\title{
A SERVICE IMPROVEMENT OPPORTUNITY FOR PHARMACIST INDEPENDENT PRESCRIBERS? A LITERATURE REVIEW EXAMINING THE RELATIONSHIP BETWEEN POOR DIABETES CONTROL AND THE CO-EXISTENCE OF MENTAL HEALTH ISSUES
}

\author{
NASREEM BIBI'1, HANA MORRISSEY1* ${ }^{*}$, PATRICK BALL1 \\ ${ }^{1}$ School of Pharmacy, University of Wolverhampton, Wulfruna Street, Wolverhampton, WV1 1LY, United Kingdom \\ Email: hana.morrissey@wlv.ac.uk
}

Received: 13 Apr 2019, Revised and Accepted: 13 Jun 2019

\begin{abstract}
Diabetes and mental illness are clinically managed by diverse pathways. However, an association between the two has been observed and evidence is growing that when poorly addressed, therapy adherence is low and outcomes are poor. To date, no intervention has been shown to provide sustained improvements in adherence, outcome, quality of life or provider cost savings. This literature review was undertaken as a foundation to a research project to examine whether there is an opportunity for current independent prescriber pharmacists, with or without further training, could as part of a primary care team, contribute their expertise to achieving better and sustainable outcomes for these conditions, where current treatment models are heavily medication-dependent. It reinforces the idea that these conditions are interlinked but by poorly understood mechanisms and suggests that a new approach is required in order to improve outcomes for this complex patient group.
\end{abstract}

Keywords: Depression, Type 2 Diabetes, Diabetes, Independent Prescribing Pharmacist, Pharmacist

(C) 2019 The Authors. Published by Innovare Academic Sciences Pvt Ltd. This is an open access article under the CC BY license (http://creativecommons.org/licenses/by/4.0/)

DOI: http://dx.doi.org/10.22159/ijcpr.2019v11i4.34930

\section{INTRODUCTION}

Mental illness and diabetes are two major conditions that negatively impact health and wellbeing. Both are currently increasing in the UK limiting the quality of life and stretching resources. Both are affected by poor adherence to therapy, and now evidence appears to be growing that there may be a link between these conditions. ${ }^{1}$ The National Institute of Clinical Excellence (NICE) has guidelines on how these conditions are managed individually, step by step. However, many studies appear to demonstrate a link with shared risk factors and disease mechanisms [1].

Diabetes mellitus (DM) and mental illness are chronic diseases having a high rate of morbidity and mortality when not managed correctly [2]. It can be divided into two major sub-types; Type 1 (DM-T1) and Type 2 (DM-T2). Both forms are increasing in frequency in the UK, with DM-T2 increasing most rapidly [3]. NICE defines DM-T2 as a chronic metabolic condition characterised by insulin resistance and insufficient pancreatic insulin production which, in turn, causes high blood glucose levels, whilst in DM-T1 there is little or no insulin produced by the pancreas [4].

Mental illness is an umbrella term including a variety disorders such as depression, anxiety, schizophrenia, psychosis, and bipolar affective disorder.

The numbers of people receiving psychological therapies for health conditions have more than doubled in the last four years ${ }^{4}$. DM has also been rapidly increasing, especially DM-T2 [5]. It is associated with long term complications, reduced quality of life and life expectancy $[4,6]$. Both conditions have a modifying effect on the prognosis of the other despite separate aetiology and management. To date, an ideal strategy for the management of patients with mental health and chronic disease comorbidity has not been elucidated.

\section{Aims and objectives}

The growth in the number of pharmacist independent prescribers in the UK creates opportunities to revisit models of care, and identify new ways of exploiting all the skills represented in the primary health workforce. This review of the literature explores the opportunities for future research to optimize the management of patients with co-morbid depression and diabetes, and whether potential exists for the expertise of the pharmacist to be applied to improve adherence and outcomes.

\section{Study questions}

Can improving mental health through treating mental illnesses improve diabetes control in those who have it?

Is there evidence that effectively treating mental illness improves patient adherence to therapy and accordingly improves diabetes control through changed self-care behaviour? Is this an area where independent prescribing pharmacists working as part of a primary care team can utilise their skills to help to optimize outcomes.

\section{Literature search method and search criteria}

The search strategy used PubMed, Science Direct, and Google Scholar. Specific keywords used to refine the searches were $<$ Mental illness>, <mental ill health>, <diabetes>, <Type 2 diabetes>, $<$ diabetes mellitus $>$ and $\left\langle\mathrm{Hba}_{1} \mathrm{c}\right\rangle$. First search produced over 300,000 articles, when we applied filters (published 2005 onwards, published in English) there were 12,000 items. Titles were reviewed to eliminate opinion papers, webpages and review papers. There were 500 possible papers where the title contained either of the conditions that were considered for further review. Primary studies dealing with chronic physical disease and mental illness comorbidity were selected, particularly those addressing therapy and/or medication adherence.

\section{Review findings}

In a cross-sectional study, Kruse produced evidence that the relationship between mental disorder and glycaemic control remained unclear. ${ }^{7}$ their study showed that people with diabetes were not more likely to be diagnosed with mental disorders when compared to individuals without diabetes. They found the odds ratio (OR) for anxiety disorders in patients with diabetes were higher than in the general population (OR 1.93, 95\% CI 1.19-3.19). However, the relationship between diabetes and affective disorders was not significant after controlling for age, sex, marital status and socioeconomic status. Conversely, the relationship between diabetes and anxiety disorder remained significant after controlling those variables [7]. 
Hein, in a study assessing the prevalence of risk factors in patients with DM-T2 and depression, showed a high incidence of DM-T2 in patients diagnosed with depression $(n=703)$ [8]. Their study showed that if patients with co-morbid DM-T2 and depression did not receive treatment and support for their depression as well as their diabetes, both diseases had poor outcomes. They also demonstrated that individuals with major depression have a greater risk of a sedentary lifestyle and decreased physical activity. They concluded that 'obesity, male sex and older age' are risk factors for DM-T2 with depressed patients having a poor prognosis compared to those with DM-T2 only [8].

Hein $^{8}$ also stated that 'when depression was treated in individuals with DM-T2, modifiable risk factors were better managed and their DM-T2 was better-controlled suggesting that by managing depression in diabetes, the disease burden may also be reduced due to lower cost of diabetes complications. The study demonstrates that risk factors for diabetes can easily fit into the lifestyle changes (loss of motivation, overeat or sleep, weight loss or gain), leading to or worsening DM.

Harkness $^{2}$ carried out a systematic review 'identifying psychosocial interventions that improve both physical and mental health in patients with diabetes'. Their results showed the importance of managing mental health in long term conditions and the effect mental illness has on long-term condition outcomes. Both Hein [8] and Harkness [2] concur; risk factors need to be managed and treated prophylactically to prevent deterioration of the long-term condition. Harkness showed that ' $53 \%$ of interventions focused on lifestyle alone, $29 \%$ focused on mental health and $18 \%$ on both aspects. The interventions examined included; education and cognitive behavioral therapy. ${ }^{2}$ However, the author concluded that despite the potential interaction between the two conditions, 'therapeutic pathways have proven elusive, noting that the effect long-term chronic conditions and mental illness exert on each other is not only restricted to DM-T2 but to the overall health of people with this combination of comorbidity.

Another study by Shin used a self-assessment questionnaire completed by both patients with mental illness and their treating clinicians. ${ }^{9}$ They concluded that there was a simple tool to identify people at high risk of DM-T2 in a patient with mental illness. Their study showed that from their sample of 100 participants, nine had DM-T2 and 34 were pre-diabetic. The authors explained the link between antipsychotic medications and poor glycaemic control. They further stated that typical antipsychotic medications are more likely to be associated with DM-T2 than atypical antipsychotic agents and that their tool raised awareness of the risk and prompted clinicians to look for diabetes [9].

A national population-based, open cohort study designed by Huang showed the annual incidence of mental illness in diabetic patients was significantly higher than in the general population [10]. The article showed the incidence was higher in female patients and elderly patients of low income and from rural areas when compared to all others. This study had many limitations which made the results not applicable to the UK population. It was conducted in a developing country and patients had multiple comorbidities that could have further effects on diabetes prognosis. The author questioned whether diabetes is a risk factor for mental illness or whether mental illness is a risk factor for diabetes. They concluded this remained unanswered [10].

A review by Sajatovic compared older and younger patients $(n=200)$ with serious mental illness and examined the effect of early treatment of mental illness on diabetes prognosis [11]. The study showed patients with depression had poorly controlled diabetes and more hospital emergency visits. They also found that older patients had better control than younger patients. In severe mental illness, the control of diabetes declined, and the condition became worse. In a later study, they conducted a 60-week randomized controlled trial based on a self-management intervention which focussed on training in DM-T2 management [12]. This study showed that nonpharmacological interventions can improve $\mathrm{HbA}_{1} \mathrm{c}$ in diabetic patients with mental illness through managing mental illness as a modifiable risk factor.
Gron carried out a systematic review on improving diabetes care in patients with severe mental illness [13]. The authors stated that a non-pharmacological approach in patients with mental illness using exercise, nutrition, counseling, behavior modeling and increased disease awareness to reduce $\mathrm{HbA}_{1} \mathrm{c}$, fasting plasma glucose, body mass index and weight, was effective in preventing poor control and further deterioration [13].

Belvederi-Murri concluded that glycaemic control seems to influence the severity of patients' depressive symptoms but the effect on glycaemic control improvement was insignificant [14]. The study indicated that improving DM-T2 control improved their quality of life and mental illness. This study showed that glycaemic control impacted the severity of depression due to the increasing distress related to keeping diabetes under control [14].

Telo also found that mental disorders are common in adolescents with and without DM-T1 [15]. The author stated that patients with DM-T1 should be assessed appropriately for mental health to prevent the development of mental illness.

Solanki published a literature review looking specifically for a link between bipolar affective disorder and diabetes and concluded that whilst the evidence was not conclusive patients with these comorbidities appear to have better outcomes when both issues are addressed [16].

Nasker conducted a comprehensive systematic review of the link between depression and DM [17]. The review focused on the Asian community, in particular from an Indian background. This review found a significant association between the two conditions. They reported and analyzed results from studies highlighting the potential link between the two conditions, finding that 2-4 y after diagnosis was associated with a significant increase in the risk of depression' in those patients with diabetes. This study concluded that diabetes is the cause of patients falling into depression and having diabetes is a factor for depression [17].

Another recent article on diabetes and mental health looked at the two chronic conditions in an interesting manner; the author examines how something termed 'diabetes-distress' could be the cause of the mental illness. Robinson shows how the stress of being diagnosed with diabetes can lead to stress which develops into a form of mental illness [18]. Using the studies examined, they show the term 'diabetes-distress' is used to describe 'the despondency and emotional turmoil specifically related to living with diabetes and the need for continual monitoring and treatment.

A meta-analysis by Anderson showed 'the presence of DM doubles the odds of comorbid depression' [19]. Ten patients on the controlled studies reported 'depression estimates separately, by type (DM-T1, $n=3$; DM-T2, $n=7$ ). The odds ratio for depression was significantly higher in both DM-T1 (OR $=2.9,95 \%$ CI $1.6-5.5, \chi^{2}=$ $12.8, P<0.0003)$ and DM-T2 (OR $=2.9,95 \%$ CI $2.3-3.7, \chi^{2}=84.3$, $P<0.0001)$ compared to non-diabetic control subjects.' These results suggest a significant link between the two conditions.

Ogawa looked at the factors associated with glycaemic control and diabetes self-care among outpatients with schizophrenia and DM-T2 [20]. They looked at the severity of schizophrenia on diabetes selfcare in 38 participants, through reviewing their medical records. Their study showed that there was no difference in the $\mathrm{HbA}_{1} \mathrm{c}$ level between the two groups of subjects classified by the severity of schizophrenia. It was seen that in some of the patients, diabetic control was better in those with a 'high brief psychiatric rating scale score $(\mathrm{P}<0.05)$. Dickerson focused on the knowledge of patients with DM and mental health comorbidity [21]. They studied the level of patient self-awareness of their conditions and reported on how patient education on diabetes had a beneficial effect on overall control including in those with mental illness. A trend in patient education was observed; they noted, as did Glasgow [22] that 'disease-specific diabetes knowledge may be a process or mediating variable that interacts with other factors to affect self-care, which then may affect short-and long-term health outcomes.' The statement shows education can really help in patient care of these chronic conditions. Dickerson [21] explains how variables in each condition can cause deterioration of the other. For example; comfort 
eating can lead to obesity, which in turn can affect diabetes control and worsen body image, leading to worsening of depression.

Musselman conducted a study on the relationship between depression and both types of DM; epidemiology, biology and treatment [23]. They analyzed studies to establish the relationship between mental health and diabetes finding that 'depression and its associated symptoms constitute a major risk factor in the development of DM-T2 and that it may accelerate the onset of diabetes complications.' They demonstrated that depression can lead to the development of DM-T2 and further, cause complications in the condition. Hayward echoed the same thought process in patients with mental illness, he believed that people with 'psychiatric disorders generally do have multiple risk factors for the development of DM-T2 including physical inactivity and obesity' [24].

Pendlebury examined the rising trend of diabetes alongside other health abnormalities in patients with severe mental illness [25]. The authors explained that the causes of diabetes in people with severe mental illness, as in the general population, are potentially modifiable lifestyle factors or antipsychotic medications. The key is the modifiable lifestyle changes which can improve the care of the patient. The authors explained the key area to work on this is 'healthcare professionals working in psychiatry and diabetes care need to collaborate closely to provide the integrated care'. They showed how healthcare professionals are failing in depression management is an additional link between the two conditions [25].

Fagiolini [26] shared the views of Pendlebury [25] in terms of the recognition between mental disorders and physical health complications being undertreated, resulting in further deterioration as well as other co-morbidities [26]. Under-treatment was highlighted by the authors, indicating that healthcare professionals need to implement these aspects in patients' management plans.

According to Hajek DM-T2 can itself cause an effect on the brain causing mental illness [27]. Hajek stated DM-T2 frequently co-occurs with bipolar affective disorder. This was a study on patients with DM-T2 alongside bipolar disease where they analysed a series of neuro-images. It showed a link in which poorly controlled diabetes exerted an effect on the participants' cognitive function. He concluded that 'the findings emphasize the importance of improving diabetes care in bipolar disease and suggest potential options for treatment of neuroimaging alterations' [27].

Beavers conducted a prospective cohort study on the link between the two chronic conditions [28]. They questioned whether long term glucose exposure in patients had any association with cognitive and physical function. The study included 879 participants diagnosed with DM-T2, tracked over 7-years where $\mathrm{HbA}_{1} \mathrm{c}$ was measured at regular intervals alongside tests assessing mental state. The study showed that longitudinal glucose exposure was associated with physical and cognitive function.

Convit examined the link between cognitive impairment in insulin resistance through an explanatory model approach [29]. The study explored the link between the two and explained how there is a 'consistent and growing literature reporting memory and other cognitive problems among individuals with DM as well as those with pre-diabetes.' Bjorgaas stated that the metabolic pathway in DM may directly contribute to memory impairment [30]. Some studies have shown that improving and managing diabetes by improving glucose tolerance has shown to show an effect on cognitive improvements [31].

Goldberg conducted a cross-sectional analysis on the quality of diabetes care among adults with serious mental illness [32]. The study shows lower quality of diabetes care was found in people with serious mental illness. This researcher focussed on the management of diabetes and noticed that patients diagnosed with a serious mental illness were more likely to be poorly managed, linking to further deterioration of both chronic disabling conditions [32].

In their study; Cardiovascular disease and diabetes in people with severe mental illness position statement from the European Psychiatric Association (EPA), supported by the European Association for the Study of Diabetes (EASD) and the European Society of Cardiology (ESC), De Hert et al., conducted a study on cardiovascular disease and diabetes in people with severe mental illness SMI, which looked at the various factors influencing mental illness, leading to worsening of mental health [33]. The prospective cross-sectional studies conducted in Belgium confirmed many patients are diagnosed with mental illness and diabetes at the time of their first diagnosis. They stated 'EPA, supported by the EASD and the ESC, published this statement with the aim of improving the care of patients suffering from SMI. The intention is to initiate cooperation and shared care between the different healthcare professionals and to increase the awareness amongst psychiatrists and primary care physicians caring for patients with SMI of the need to screen and treat increased cardiovascular risk factors and diabetes. In addition, the academic associations involved with this statement point out that more research is required concerning the cardiovascular problems of people with SMI and their treatment.'

A systematic review by Mclntyre showed that the prevalence of diabetes diagnosis was higher in patients with bipolar illness (3fold) than that in the general population' [34].

Ismail stated that 'adherence difficulties and psychological problems are associated with poor glycaemic control in diabetes,' in essence stating that an individual with poor diabetic control is more likely to have psychological issues potentially leading to mental illnesses [35]. The researchers examined 25 different trials and concluded that patients who are managed and receive some form of therapy i.e. psychological therapies are more likely to have better long-term glycaemic control in patients with DM-T2 [35].

A systematic review conducted by Wang looked at nonpharmacological treatment for depression and its effect on diabetes and concluded that it produces a limited effect on glycaemic control in individuals with DM-T2 [36]. Another systematic review by Baumeister looked at psychological and pharmacological interventions for depression in patients with DM and depression [37]. They concluded that; 'Psychological and pharmacological interventions have a moderate and clinically significant effect on depression outcomes in diabetes patients. Glycaemic control improved moderately in pharmacological trials, while the evidence is inconclusive for psychological interventions. Adherence to diabetic treatment regimens, diabetes complications, death from any cause, health economics and QoL have not been investigated sufficiently. Overall, the evidence is sparse and inconclusive due to several low-quality trials with a substantial risk of bias and the heterogeneity of examined populations and interventions.

Lunghi confirmed the link between depression and poor medication adherence in patients with DM-T2 comorbidity and that those patients might benefit from adherence-enhancing interventions [38]. They reported that depression was higher in patients with diabetes than the population without diabetes and that complication arose in those patients that suffered mental illness. The authors also explained that younger women were at a higher risk.

A systematic review conducted by Gorczynski [39] shared similar views to Lunghi [40]. This systematic review showed that medication adherence is important in the diabetic patient with comorbid schizophrenia. These vulnerable patients were seen to deteriorate in both conditions if adherence was low. Gorzynski also highlighted the vulnerability of female young patients compared to the rest of the population [39].

Gentil looked at the complications of the two chronic conditions from a different angle [41]. The authors looked at adherence to oral antidiabetic agents among older adults with mental disorders and the effect on health care costs due to disease poor control. Gentil stated that people with DM-T2 are twice as likely to have anxiety and depression when compared to general population [41]. Similarly, in the Gonzalez meta-analysis, it was explained that depression can worsen self-care, but depended on the type of self-care measured [42]. The study showed that patients with mental illness missed their DM medical appointments more than those in the general population.

\section{CONCLUSION}

This review further confirms the finding of others; an association between mental illness and diabetes is common, as is association between mental illness and other chronic diseases. Evidence 
supporting a causal relationship is mixed, but the problems need to be addressed. To date, the interventions attempted lack evidence of achieving major changes in medication adherence, clinical outcomes or provider cost-savings. However, he interventions attempted mostly have treated adherence to therapy as one-dimensional; the patient is either adherent to their therapy or they are not. To facilitate the interpretation of findings, future research will require tighter definitions of the mental health issues and co-morbidities addressed. In addition, adherence must be managed as a multidimensional issue with intentional and non-intentional components.

Effectively addressing such issues adequately in primary care would also need to move away from presenting problem focussed 10minute appointments; comprehensively addressing the needs of this group will require a change of approach to primary care.

\section{BENEFIT TO PHARMACY PRACTICE AND FUTURE RESEARCH}

This literature review was conducted to inform the development of a research study to aiming to utilize the skills of independent prescriber pharmacists within primary care teams within a treatment model for patients with diabetes/depression co-morbidity that can be evaluated to see if it is able to deliver sustainable benefits and improved treatment outcomes.

\section{AUTHORS CONTRIBUTIONS}

All the author have contributed equally

\section{CONFLICT OF INTERESTS}

Declare none

\section{REFERENCES}

1. Charles EF, Lambert CG, Kerner B. Bipolar disorder and diabetes mellitus: evidence for disease-modifying effects and treatment implications. Int J Bipolar Disord 2016;4:13.

2. Harkness E, MacDonald W, Valderas J, Coventry P, Gask L, Bower P. Identifying psychosocial interventions that improve both physical and mental health in patients with diabetes a systematic review and meta-analysis. Diabetes Care 2010;33:926-30.

3. NICE Guideline NG18. Diabetes (type 1 and type 2) children and young people: diagnosis and management. National Institute for Health and Care Excellence; 2016. Available from: https://www.nice.org.uk/guidance/ng18/chapter/Introductio n. [Last accessed on 10 Oct 2018]

4. NICE guidance and current practice report. Mental Health. National Institute for Health Care Excellence UK; 2017. Available from: https://webcache.googleusercontent.com/search?q=cache:vE98m Z1x9SUJ: https://www.nice.org.uk/media/default/about/whatwe-do/into-practice/measuring-uptake/nice-guidance-andcurrent-practice-report-mental-health.

pdf+andcd=1 andhl=enandct=clnkandgl=uk. [Last accessed on 10 Oct 2018]

5. Beka Q, Bowker S, Savu A, Kingston D, Johnson AJ, Kaul P. Development of perinatal mental illness in women with gestational diabetes mellitus: a population-based cohort study. Can J Diabetes 2018;42:350-5.

6. NICE Guidelines NG28 Type 2 Diabetes in adult management. National Institute for Health Care Excellence UK; 2017. Available from:https://www.nice.org.uk/guidance/ng28/chapter/Introducti on [Last accessed on 10 Oct 2018]

7. Kruse J, Schmitz N, Thefeld W. On the association between diabetes and mental disorders in a community sample. Diabetes Care 2003;26:1841-6.

8. Hein M, Lanquart JP, Loas G, Hubain P, Linkowski P. Prevalence and risk factors of type 2 diabetes in major depression: a study on 703 individuals referred to sleep examination. Psychosomatics 2018;59:144-57.

9. Shin JK, Shortridge Baggett LM, Sachmechici I, Barron C, Chiu YL, Bajracharya B, et al. Screening for type 2 diabetes mellitus in patients with mental illness: application of a self-assessment score for diabetes mellitus risk. Psychiatry Res 2014;220:1037-42.

10. Huang CJ, Wang SY, Lee MH, Chiu HC. Prevalence and incidence of mental illness in diabetes: a national population-based cohort study. Diab Res Clin Pract 2011;93:106-14.
11. Sajatovic M, Gunzler D, Einstadter D, Thomas C, McCormick R, Perzynski AT, et al. A preliminary analysis of individuals with serious mental illness and comorbid diabetes. Arch Psychiatr Nurs 2016;30:226-9.

12. Sajatovic M, Gunzler DD, Kanuch SW, Cassidy KA, Tatsuoka C, McCormick R, et al. A 60-week prospective RCT of a selfmanaged intervention for individuals with serious mental illness and diabetes mellitus. Psychiatr Serv 2017;68:883-90.

13. Gron AO, Dalsgaard EM, Ribe AR, Seidu S, Mora G, Cebrian Cuenca AM, et al. Improving diabetes care among patients with severe mental illness: a systematic review of the effect of an intervention. Primary Care Diabetes 2018;12:289-304.

14. Belvederi Murri M, Mamberto S, Briatore L, Mazzucchelli C, Amore M, Cordera R. The interplay between diabetes, depression and affective temperaments: a structural equation model. J Affect Disord 2017;219:64-71.

15. Telo GH, Cureau FV, Lopes CS, Schaan BD. Common mental disorder in adolescents with and without type 1 diabetes: reported occurrence from a countrywide survey. Diabetes Res Clin Pract 2017;135:192-8.

16. Solanki V, Ball PA, Morrissey H. Disease-modifying the effect of type 2 diabetes mellitus and bipolar disorder comorbidity. Int J Curr Med Pharm Res 2018;4:2253-3261.

17. Nasker S, Victor R, Nath K. Depression in diabetes mellitus-A comprehensive systematic review of literature from an Indian perspective. Asian J Psychiatr 2017;27:85-100.

18. Robinson DJ, Coons M, Haensel H, Vallis M, Yale JF. Diabetes and mental health. Diabetes canada clinical practice guidelines expert committee. Can J Diabetes 2018;42(s1):S130-S141.

19. Anderson RJ, Freedland KE, Clouse RE, Lustman PJ. The prevalence of comorbid depression in adults with diabetes: a meta-analysis. Diabetes Care 2001;24:1069-78.

20. Ogawa M, Miyamoto Y, Kawakami N. Factors associated with glycemic control and diabetes self-care among outpatients with schizophrenia and type 2 diabetes. Arch Psychiatr Nurs 2011;25:63-73.

21. Dickerson FB, Ricard MPH, Goldberg W, Brown CH, Krevenbuhl JA, Wohlheiter KMS, et al. Diabetes knowledge among persons with serious mental illness and type 2 diabetes. Psychosomatics 2005;46:418-24.

22. Glasgow RE. Outcomes of and for diabetes education research. Diabetes Educ 1999;25(Suppl 6):74-88.

23. Musselman DL, Betan E, Larsen H, Phillips LS. Relationship of depression to diabetes types 1 and 2 epidemiology, biology and treatment. Biol Psychiatry 2003;54:317-29.

24. Hayward C. Psychiatric illness and cardiovascular disease risk. Epidemiol Rev 1995;17:129-38.

25. Pendlebury J, Holt R. Managing diabetes in people with severe mental illness. J Diabetes Nurs 2010;14:328-39.

26. Fagiolini A, Goracci A. The effects of undertreated chronic medical illnesses in patients with severe mental disorders. J Clin Psychiatry 2009;70(Suppl 3):22-9.

27. Hajek T, Calkin C, Blagdon R, Slaney C, Alda M. Type 2 diabetes mellitus: a potentially modifiable risk factor for neurochemical brain changes in bipolar disorders. Biol Psychiatr 2015;77:295303.

28. Beavers K, Leng I, Rapp S, Miller M, Houston D, Marsh A, et al. Effects of longitudinal glucose exposure on cognitive and physical function: results from the action for health in diabetes movement and memory study. J Am Geriatr Soc 2016;65:137-45.

29. Convit A. Links between cognitive impairment in insulin resistance: an explanatory model. Neurobiol Aging 2005;26(s1):31-5.

30. Bjorgaas M, Gimse R, Vik T, Sand T. Cognitive function in type 1 diabetic children with and without episodes of severe hypoglycaemia. Acta Pediatr 1997;86:148-53.

31. Gradman TJ, Laws A, Thompson LW, Reaven GM. Verbal learning and/or memory improves with glycemic control in older subjects with non-insulin-dependent diabetes mellitus. J Am Geriatr Soc 1993;41:1305-12.

32. Goldberg RW, Kreyenbuhl JA, Medoff DR, Dickerson FB, Wohlheiter K, Fang LJ, et al. Quality of diabetes care among adults with serious mental illness. Psychiatr Serv 2007;58:53643. 
33. De Hert M, Dekker JM, Wood D, Kahl KG, Holt RI, MöllerHJ. Cardiovascular disease and diabetes in people with severe mental illness position statement from the European Psychiatric Association (EPA), supported by the European Association for the Study of Diabetes (EASD) and the European Society of Cardiology (ESC). Eur Psychiatry 2009;24:412-24

34. Mclyntyre RS, KonarskiJ Z, Misener VL, Kennedy SH. Bipolar disorder and diabetes mellitus: epidemiology, aetiology and treatment implication. Ann Clin Psychiatry 2005;17:83-93.

35. Ismail K, Winkley K, Rabe Hesketh S. Systematic review and meta-analysis of randomised controlled trials of psychological interventions to improve glycaemic control in patients with type 2 diabetes. Lancet 2004;363:1589-97.

36. Wang MY, Tsai PS, Chou KR, Chen CM. A systematic review of the efficacy of non-pharmacological treatments for depression on glycaemic control in type 2 diabetics. J Clin Nurs 2008; $17: 2524-30$

37. Baumeister $\mathrm{H}$, Hutter $\mathrm{N}$, Bengel J. Psychological and pharmacological interventions for depression in patients with diabetes mellitus and depression. Cochrane Database Systematic Rev 2012;12:CD008381.

38. Lunghi C, Zongo A, Moison J, Gregoire JP, Guenette L. The impact of incident depression on medication adherence in patients with type 2 diabetes. Diabetes Metab 2017;43:521-8.

39. Gorczynski P, Patel H, Ganguli R. Adherence to diabetes medication in individuals with schizophrenia: a systematic review of rates and determinants of adherence. Clin Schizophre Relat Psychoses 2017;10:191-200.

40. Lunghi C, Moisan J, Gregoire JP, Guenette L. Incidence of depression and associated factors in patients with type 2 diabetes in quebec, canada: a population-based cohort study. Medicine (Baltimore) 2016;95:e3514.

41. Gentil L, Vasiliadi HM, Preille M, Berbiche D. Adherence to oral antihyperglycemic agents among older adults with mental disorders and its effect on health care costs, Quebec, Canada 2005-2008. Prev Chronic Dis 2015;12:E230.

42. Gonzalez JS, Peyrot M, McCarl LA, Collins EM, Matthews LS, Mimiaga J, et al. Depression and diabetes treatment nonadherence: a meta-analysis. Diabetes Care 2008;31:2398-403. 